Commun.Fac.Sci.Univ.Ank.Series A 1

Volume 61, Number 2, Pages 17-34 (2012)

ISSN $1303-5991$

\title{
SOME BASIC RESULTS ON THE SETS OF SEQUENCES WITH GEOMETRIC CALCULUS
}

\author{
CENGİZ TÜRKMEN AND FEYZİ BAŞAR
}

\begin{abstract}
As an alternative to the classical calculus, Grossman and Katz [Non-Newtonian Calculus, Lee Press, Pigeon Cove, Massachusetts, 1972] introduced the non-Newtonian calculus consisting of the branches of geometric, anageometric and bigeometric calculus. Following Grossman and Katz, we construct the field $\mathbb{C}(G)$ of geometric complex numbers and the concept of geometric metric. Also we give the triangle and Minkowski's inequalities in the sense of geometric calculus. Later we respectively define the sets $\omega(G), \ell_{\infty}(G)$, $c(G), c_{0}(G)$ and $\ell_{p}(G)$ of all, bounded, convergent, null and $p$-absolutely summable sequences, in the sense of geometric calculus and show that each of the set forms a vector space on the field $\mathbb{C}(G)$ and a complete metric space.
\end{abstract}

\section{Preliminaries, Background and Notation}

During the renaissance many scholars, including Galileo, discussed the following problem: Two estimates, 10 and 1000, are proposed as the value of a horse, which estimates, if any, deviates more from the true value of 100? The scholars who maintained that deviations should be measured by differences concluded that the estimate of 10 was closer to the true value. However, Galileo eventually maintained that the deviations should be measured by ratios, and he concluded that two estimates deviated equally from the true value.

From the story, the question comes out this way what if we measure by ratios? The answer is the main idea of non-Newtonian calculus which is consist of many calculuses such as; the classical, geometric, ana-geometric, bi-geometric calculus. Even you can create your own calculus by choosing generator different function. Although all arithmetics are structurally equivalent, only by distinguishing among

Received by the editors June 26, 2012, Accepted: Nov. 27, 2012.

2010 Mathematics Subject Classification. 26A06, 11U10, 08A05.

Key words and phrases. Geometric calculus, algebraic structures with respect to geometric calculus, geometric sequence spaces.

The main results of this paper were presented in part at the International Conference On Applied Analysis and Algebra to be held on 29-30 June and 1-2 July 2011 in İstanbul, Turkey at the Yıldız Technical University. 
them do we obtain suitable tools for constructing all the non-Newtonian calculus. But the usefulness of arithmetics is not limited to the construction of calculus; we believe there is a more fundamental reason for considering alternative arithmetics: They may also be helpful in developing and understanding new systems of measurement that could yield simpler physical laws.

Bashirov et al. [1] have recently emphasized on the non-Newtonian calculus and gave the results with applications corresponding to the well-known properties of derivative and integral in the classical calculus. Quite recently, Uzer [7] has extended the multiplicative calculus to the complex valued functions and interested in the statements of some fundamental theorems and concepts of multiplicative complex calculus, and demonstrated some analogies between the multiplicative complex calculus and classical calculus by theoretical and numerical examples. Bashirov and Rıza [2] have studied on the multiplicative differentiation for complex-valued functions and established the multiplicative Cauchy-Riemann conditions. Bashirov et al. [3] have investigated various problems from different fields can be modeled more efficiently using multiplicative calculus, in place of Newtonian calculus. Quite recently, Çakmak and Başar [4] have emphasized that each of the set $\omega(\mathbb{N}), \ell_{\infty}(\mathbb{N})$, $c(\mathbb{N}), c_{0}(\mathbb{N})$ and $\ell_{p}(\mathbb{N})$ of all, bounded, convergent, null and $p$-absolutely summable sequences forms a vector space on the field $\mathbb{R}(\mathbb{N})$ of non-Newtonian real numbers and a complete metric space.

Geometric calculus is an alternative to the usual calculus of Newton and Leibniz. It provides differentiation and integration tools based on multiplication instead of addition. Every property in Newtonian calculus has an analog in multiplicative calculus. Generally speaking, multiplicative calculus is a methodology that allows one to have a different look at problems which can be investigated via calculus. In some cases, for example for growth related problems, the use of multiplicative calculus is advocated instead of a traditional Newtonian one.

The set $\mathbb{R}(G)$ of positive real numbers are defined by $\mathbb{R}(G):=\left\{e^{x}: x \in \mathbb{R}\right\}:=$ $\mathbb{R}^{+} \backslash\{0\}$. Throughout this document, geometric calculus is denoted by (GC) and classical calculus is denoted by (CC). A generator is one-to-one function whose domain is $\mathbb{C}$ and whose range is a subset of $\mathbb{C}$, the set of complex numbers. As a generator, we choose the function exp from $\mathbb{C}_{s t r}$ to the set $\mathbb{C}(G):=\mathbb{C} \backslash\{0\}$, (see figure below), that is to say that 


$$
\begin{aligned}
\alpha: \mathbb{C}_{s t r} & \longrightarrow \mathbb{C}(G) \\
z & \longmapsto \alpha(z)=e^{z}=w
\end{aligned} \text { and } \begin{array}{rlll}
\alpha^{-1}: & \mathbb{C}(G) & \longrightarrow & \mathbb{C}_{s t r} \\
w & \longmapsto & \alpha^{-1}(w)
\end{array}
$$

(a)

where

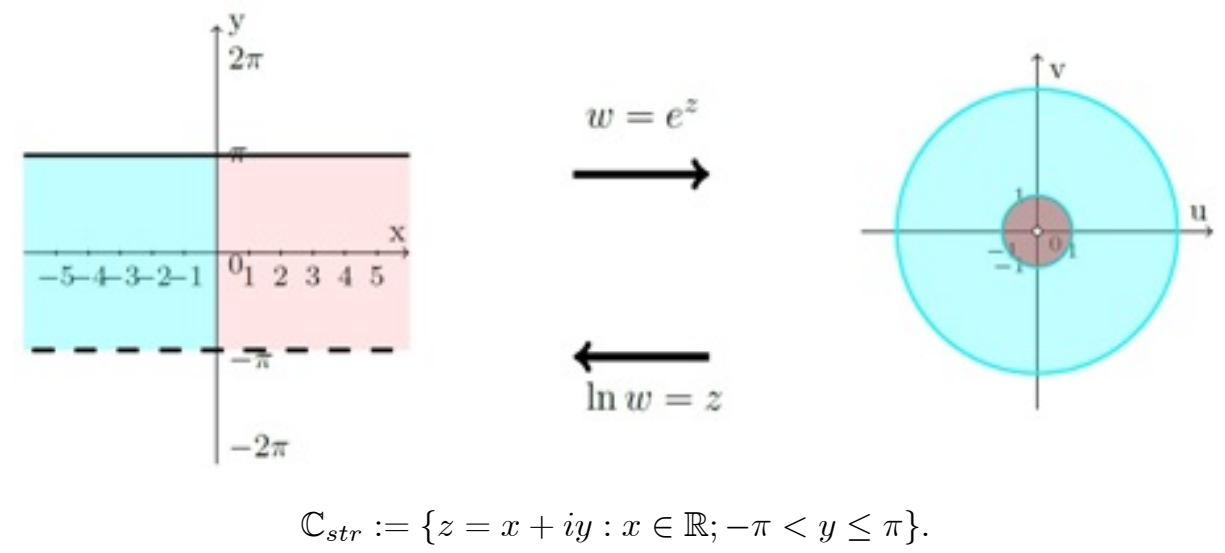

In this situation, geometric zero is $e^{0}=1$ and geometric one is $e^{1}=e$. Similarly, the set $\mathbb{Z}(G)$ of geometric integers is given by $\mathbb{Z}(G)=:\left\{e^{m}: m \in \mathbb{Z}\right\}$, where $\mathbb{Z}$ denotes the set of integers.

Remark 1.1. Since it is known that the base $e$ of natural logarithm is transcendental, one can easily see that $e \in \mathbb{Z}(G)$ but $e \notin \mathbb{Z} \backslash\{0\}$. This reveals that $\mathbb{Z}(G) \neq \mathbb{Z} \backslash\{0\}$.

We define the set $\mathbb{C}(G)$ of geometric complex numbers, as follows:

$$
\begin{aligned}
\mathbb{C}(G):= & \left\{w=u \oplus i_{g} \odot v: u, v \in \mathbb{R}(G) \text { and } i_{g}=\sqrt{1 \ominus e}^{G}\right\} \\
= & \left\{e^{z}=e^{x} \cdot\left(e^{y}\right)^{\ln e^{i}}: e^{x}, e^{y} \in \mathbb{R}(G) \text { and } e^{i}=e^{\sqrt{-1}}\right\} \\
= & \left\{e^{x+i y}: x \in \mathbb{R} ;-\pi<y \leq \pi \text { and } i=\sqrt{-1}\right\} \\
= & \left\{e^{z}: z \in \mathbb{C}_{s t r}\right\} .
\end{aligned}
$$

It is easy to see that $\mathbb{C}(G)=\mathbb{C} \backslash\{0\}$. It is clear by the definition of complex $\exp$ function that $\alpha(z)=e^{z} \neq 0$ for all $z \in \mathbb{C}_{s t r}$. Since $\alpha$-generator is a bijective function, it maps all complex numbers without zero to the range set. Each generator generates exactly one arithmetic, and conversely each arithmetic is generated by exactly one generator.

A complete field is a system consisting of a set A and four binary operations $\oplus, \ominus, \odot, \oslash$. We call $A$ the realm of complete field. By an arithmetic, we mean complete field whose realm is a subset of $\mathbb{C}$. 
Consider any generator $\alpha$ with range $A \subseteq \mathbb{C}$. By $\alpha$-arithmetic we mean the arithmetic whose realm is $A$ and whose operations are defined as follows: For $z, w \in \mathbb{C}$ and any generator $\alpha$,

$$
\begin{aligned}
\alpha \text {-addition : } & z \oplus w=\alpha\left\{\alpha^{-1}(z)+\alpha^{-1}(w)\right\} \\
\alpha-\text { subtraction }: & z \ominus w=\alpha\left\{\alpha^{-1}(z)-\alpha^{-1}(w)\right\} \\
\alpha-\text { multiplication }: & z \odot w=\alpha\left\{\alpha^{-1}(z) \times \alpha^{-1}(w)\right\} \\
\alpha-\text { division }: & z \oslash w=\alpha\left\{\alpha^{-1}(z) \div \alpha^{-1}(w)\right\}
\end{aligned}
$$

Particularly if we choose the $\alpha$-generator as the identity function then $\alpha(z)=z$ for all $z \in \mathbb{C}$ which implies that $\alpha^{-1}(z)=z$, that is to say that $\alpha$-arithmetic is reduced to the classical arithmetic.

$$
\begin{aligned}
& \alpha \text {-addition : } z \oplus w=\alpha\left\{\alpha^{-1}(z)+\alpha^{-1}(w)\right\}=\alpha\{z+w\}=z+w \quad: \quad \text { classical addition } \\
& \alpha \text {-subtraction : } z \ominus w=\alpha\left\{\alpha^{-1}(z)-\alpha^{-1}(w)\right\}=\alpha\{z-w\}=z-w \quad: \quad \text { classical subtraction } \\
& \alpha \text {-multiplication }: z \odot w=\alpha\left\{\alpha^{-1}(z) \times \alpha^{-1}(w)\right\}=\alpha\{z \times w\}=z \times w \quad: \quad \text { classic multiplication } \\
& \alpha \text {-division : } z \oslash w=\alpha\left\{\alpha^{-1}(z) \div \alpha^{-1}(w)\right\}=\alpha\{z \div w\}=z \div w \quad: \quad \text { classical division }
\end{aligned}
$$

If we choose the $\alpha$-generator as $\exp$, then $\alpha(z)=e^{z}$ for all $z \in \mathbb{C}$ which gives that $\alpha^{-1}(z)=\ln z$, that is to say that $\alpha$-arithmetic is reduced to the Geometric arithmetic.

$$
\begin{array}{rll}
\alpha \text {-addition : } z \oplus w=\alpha\left\{\alpha^{-1}(z)+\alpha^{-1}(w)\right\}=e^{\{\ln z+\ln w\}}=z \cdot w & : & \text { geometric addition } \\
\alpha-\text { subtraction : } z \ominus w=\alpha\left\{\alpha^{-1}(z)-\alpha^{-1}(w)\right\}=e^{\{\ln z-\ln w\}}=z \div w & : & \text { geometric subtraction } \\
\alpha-\text { multiplication : } z \odot w=\alpha\left\{\alpha^{-1}(z) \times \alpha^{-1}(w)\right\}=e^{\{\ln z \times \ln w\}}=z^{\ln w} & : & \text { geometric multiplication } \\
\alpha-\text { division : } z \oslash w=\alpha\left\{\alpha^{-1}(z) \div \alpha^{-1}(w)\right\}=e^{\{\ln z \div \ln w\}}=z^{\frac{1}{\ln w}} & : & \text { geometric division }
\end{array}
$$

Now we express signs and their equivalent forms in classical calculus by the following table:

Following Bashirov et al. [1] and Uzer [7], the main purpose of this paper is to construct the classical sequence spaces with respect to geometric calculus over complex numbers. To do this, we need some preparatory knowledge about geometric calculus.

The rest of the paper is organized, as follows:

In Section 2, it is showed that the set $\mathbb{C}(G)$ of geometric complex numbers forms a field with the binary operations addition $\oplus$ and multiplication $\odot$. Further, the geometric exponent, surd and absolute value are defined and their some properties are given. Section 3 is devoted to the vector spaces over the field $\mathbb{C}(G)$ of geometric complex numbers. Additionally, some required inequalities are presented in the sense of geometric calculus and the concepts of geometric metric and geometric norm are introduced. In Section 4, subsequent to giving the corresponding results for the sequences of geometric complex numbers concerning the convergent sequences of real or complex numbers, it is stated and proved that the $n$-dimensional geometric complex space $\mathbb{C}^{n}(G)$ is a complete metric space. In Section 5 , prior to showing the sets $\omega(G), \ell_{\infty}(G), c(G), c_{0}(G)$ and $\ell_{p}(G)$ of all, bounded, convergent, null and absolutely $p$-summable sequences are the complete metric spaces it is proved that each of those sets forms a vector space over the field $\mathbb{C}(G)$ with respect 


\begin{tabular}{|c|c|c|}
\hline Name of signs & Expression in $(G C)$ & Equivalent form in (CC) \\
\hline geometric zero & 1 & $e^{0}$ \\
\hline geometric unity & $e$ & $e^{1}$ \\
\hline geometric complex number & $z$ & $e^{x}$ for $x \in \mathbb{C}_{s t r}$ \\
\hline geometric absolute value & $|z|_{G}$ & $e^{|x|}$ \\
\hline geometric imaginer & $i_{g}=\sqrt{1 \ominus e}^{G}$ & $e^{i}$ \\
\hline geometric complex number & $z=u \oplus\left(i_{g} \odot v\right)$ & $e^{m} \cdot\left(e^{n}\right)^{\ln e^{i}} ; m, n \in \mathbb{R}$ \\
\hline geometric addition & $z \oplus w$ & $e^{x} \cdot e^{y}$ \\
\hline geometric subtraction & $z \ominus w$ & $e^{x} / e^{y}$ \\
\hline geometric multiplication & $z \odot w$ & $\left(e^{x}\right)^{\ln e^{y}}$ \\
\hline geometric division & $z \oslash w$ or $\frac{z}{w} G$ & $\left(e^{x}\right)^{\frac{1}{\ln e^{y}}}$ \\
\hline geometric summation & $\sum_{G}$ & $\Pi$ \\
\hline geometric epsilon & $\varepsilon$ & $e^{\varepsilon}$ \\
\hline geometric exponent & $z^{p_{G}}$ & $\left(e^{x}\right)^{\ln p-1} e^{x}$ \\
\hline geometric surd & $\sqrt[p]{z}^{G}$ & $e^{\left(\ln e^{x}\right)^{\frac{1}{p}}}$ \\
\hline geometric ordering & $<_{G}, \leq_{G},>_{G}, \geq_{G}$ & $<, \leq,>, \geq($ identical with $(\mathrm{CC}))$ \\
\hline geometric limit & $G \lim _{n \rightarrow \infty} z_{n}$ & $\lim _{n \rightarrow \infty} e^{x_{n}}=e^{\lim _{n \rightarrow \infty} x_{n}}$ (identical with $(\mathrm{CC})$ ) \\
\hline geometric supremum & $\sup _{n}\left|z_{n}\right|_{G}$ & $\left(e^{\sup _{n}\left|x_{n}\right|}\right)($ identical with $(\mathrm{CC}))$ \\
\hline
\end{tabular}

TABLE 1. Notation in (GC) and equivalent form in (CC)

to the algebraic operations addition $\oplus$ and scalar multiplication $\odot$. In the final section of the paper, we note the sicnificance of the geometric calculus and record some further suggestions.

In the foundations of science (formerly titled, Physics: The Elements), Norman Robert Campbell, a pioneer in the theory of measurement, clearly recognized that alternative arithmetics might be useful in science, for he wrote, we must recognize the possibility that a system of measurement may be arbitrary otherwise than in the choice of unit; there may be arbitrariness in the choice of process of addition, $[5]$.

We should know that all concept in classical arithmetic have natural counterparts in $\alpha$-arithmetic. For instance; $\alpha$-zero and $\alpha$-one turn out to be $\alpha(0)$ and $\alpha(1)$. Similarly the $\alpha$-integers turn out to be the following:

$$
\ldots, \alpha(-3), \alpha(-2), \alpha(-1), \alpha(0), \alpha(1), \alpha(2), \alpha(3), \ldots
$$

Particularly by choosing generator $\alpha(x)=e^{x}$ then the set $\mathbb{Z}(G)$ of geometric integers and the set $\mathbb{R}(G)$ of geometric real numbers are given by

$$
\begin{array}{ll}
\mathbb{Z}(G)=: & \left\{e^{x}: \quad x \in \mathbb{Z}\right\}=\mathbb{Z} \backslash\{0\}, \\
\mathbb{R}(G)=: & \left\{e^{x}: \quad x \in \mathbb{R}\right\}=\mathbb{R}^{+} \backslash\{0\} .
\end{array}
$$




\section{Geometric Complex Field and Related Properties}

In the present section, we construct the geometric complex field $\mathbb{C}(G)$ and give its some properties.

Define the binary operations $\oplus$ addition and $\odot$ multiplication on the set $\mathbb{C}(G)$ of geometric complex numbers by

$$
\begin{aligned}
\oplus: \mathbb{C}(G) \times \mathbb{C}(G) & \longrightarrow \mathbb{C}(G) \\
(z, w) & \longmapsto z \oplus w=z \cdot w \\
\odot: \mathbb{C}(G) \times \mathbb{C}(G) & \longrightarrow \mathbb{C}(G) \\
(z, w) & \longmapsto z \odot w=z^{\ln w}=w^{\ln z}
\end{aligned}
$$

for all $z, w \in \mathbb{C}(G)$. Then we have:

Theorem 2.1. $(\mathbb{C}(G), \oplus, \odot)$ is a field.

Proof. Since one can easily observe by routine verification that

(i) $(\mathbb{C}(G), \oplus)$ is an Abelian group,

(ii) $(\mathbb{C}(G) \backslash\{1\}, \odot)$ is an Abelian group,

(iii) The operation $\odot$ is distributive over the operation $\oplus$, we conclude that $(\mathbb{C}(G), \oplus, \odot)$ is a field.

Now, we emphasize on the concepts of geometric exponent, surd and absolute value.

Let $x \in \mathbb{C}(G)$. Then, $x$-square, $x$-cube and $p$ th power of $x$ are defined in the sense of $(\mathrm{GC})$ as follows:

$$
\begin{aligned}
x^{2_{G}} & =x \odot x=x^{\ln x} \\
x^{3_{G}} & =x \odot x \odot x=\left(x^{\ln x}\right)^{\ln x}=x^{\ln ^{2} x} \\
& \vdots \\
x^{p_{G}} & =\underbrace{x \odot x \odot x \odot \ldots \odot x}_{p \text {-times }}=\left(\left(\left(\left(x^{\ln x}\right)^{\ln x}\right)^{\ln x}\right)^{\cdots}\right)^{\ln x}=x^{\ln ^{p-1} x}
\end{aligned}
$$

As a consequence of the concept geometric square $x^{2_{G}}$ of $x \in \mathbb{C}(G)$, we define the geometric square root $\sqrt{\cdot}^{G}$ by

$$
x=\sqrt{y}^{G}=e^{(\ln y)^{1 / 2}} \text { if } y=x^{2_{G}}=x^{\ln x} .
$$

Since $x \in \mathbb{C}(G)$ with $x=e^{y}$ for $y \in \mathbb{C}_{s t r}$, we have

$$
\begin{aligned}
{\sqrt{x^{2 G}} G}^{G} e^{\left(\ln x^{2} G\right)^{1 / 2}} & =e^{\left[\ln \left(x^{\ln x}\right)\right]^{1 / 2}} \\
& =e^{\left[\left(\ln e^{y}\right)^{2}\right]^{1 / 2}}=e^{\sqrt{y^{2}}}=e^{|y|}=\left|e^{y}\right|_{G}=|x|_{G}
\end{aligned}
$$

which gives the following property about geometric surd:

$$
{\sqrt{x^{2_{G}}}}^{G}=|x|_{G}
$$


Similarly, we define $p$ th root of geometric surd, as follows: Let $x \in \mathbb{C}(G)$ with $x=e^{y}$ and $y \in \mathbb{C}_{s t r}$. Then, $p$ th root $\sqrt[p]{x^{p_{G}}} G$ is defined by

$$
\begin{aligned}
& \sqrt[p]{x^{p_{G}} G}=e^{\left(\ln x^{p_{G}}\right)^{\frac{1}{p}}}=e^{\left[\ln \left(x^{\ln ^{(p-1)} x}\right)\right]^{\frac{1}{p}}} \\
& =e^{\left[(y)^{p}\right]^{\frac{1}{p}}}=e^{p p y^{p}}=\left\{\begin{array}{cl}
e^{|y|}=|x|_{G} & , \quad \text { if } p \text { even, } \\
e^{y}=x & , \quad \text { if } p \text { odd. }
\end{array}\right.
\end{aligned}
$$

$\alpha$-absolute value $|x|_{\alpha}$ of $x \in A \subseteq \mathbb{R}$ is defined by

$$
|x|_{\alpha}=\left\{\begin{array}{ccc}
x & , & x>\alpha(0), \\
\alpha(0) & , & x=\alpha(0), \\
\alpha(0) \boxminus x & , & x<\alpha(0),
\end{array}\right.
$$

and $\alpha$-distance is given by $\left|x_{1} \boxminus x_{2}\right|_{\alpha}$ for all $x_{1}, x_{2} \in A$. Particularly choose $I$ as $\alpha$-generator, we obtain classical absolute value $|x|$ of $x \in \mathbb{R}$ is defined, as usual, by

$$
|x|=\left\{\begin{aligned}
x & , \quad x>0 \\
0 & , \quad x=0 \\
-x \quad & x<0
\end{aligned}\right.
$$

and classical distance is given by $\left|x_{1}-x_{2}\right|$ for all $x_{1}, x_{2} \in \mathbb{R}$.

Similarly, if we choose exp as $\alpha$-generator, then we derive the geometric absolute value $|x|_{G}$ of $x \in \mathbb{R}(G)$ with $x=e^{y}$ is defined by

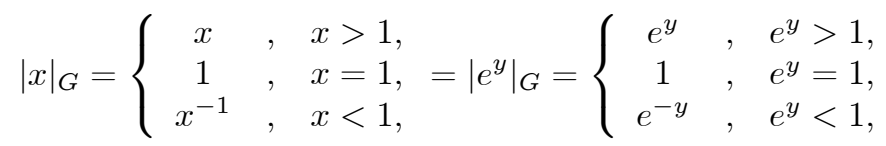

and geometric-distance is given by $\left|x_{1} \ominus x_{2}\right|_{G}=\left|x_{1} / x_{2}\right|_{G}$ for all $x_{1}, x_{2} \in \mathbb{R}(G)$ or $e^{\left|y_{1}-y_{2}\right|}=\left|e^{y_{1}} / e^{y_{2}}\right|_{G}$ for all $y_{1}, y_{2} \in \mathbb{R}$. Now, we can list some properties of geometric absolute value:

1) $u \in \mathbb{R}(G)$ with $u>1$ is called as geometric positive number. Also, $u \in \mathbb{R}(G)$ with $u<1$ is called as geometric negative number. $u \in \mathbb{R}(G)$ with $u=1$ is called as geometric unsigned number.

2) For $x_{1}, x_{2} \in \mathbb{R}(G)$ with $x_{1}=e^{y_{1}}, x_{2}=e^{y_{2}}$ it is immediate that

$$
\begin{aligned}
\left|x_{1} \ominus x_{2}\right|_{G} & =\left|x_{1} / x_{2}\right|_{G}=\left|e^{y_{1}} / e^{y_{2}}\right|_{G}=\left|e^{y_{1}-y_{2}}\right|_{G}=e^{\left|y_{1}-y_{2}\right|} \\
& =e^{\left|y_{2}-y_{1}\right|}=\left|e^{y_{2}} / e^{y_{1}}\right|_{G}=\left|x_{2} / x_{1}\right|_{G}=\left|x_{2} \ominus x_{1}\right|_{G} .
\end{aligned}
$$

That is to say that $|\cdot|_{G}$ is also symmetric with respect to subtraction.

3) In (CC), it is known that $|x \cdot y|=|x| \cdot|y|$ for all $x, y \in \mathbb{C}$. Now, we are going to give the corresponding equality in (GC). For $u, v \in \mathbb{C}(G)$ with $e^{t}=u, e^{z}=v$, since

$$
\begin{aligned}
|u \odot v|_{G} & =\left|u^{\ln v}\right|_{G}=\left|\left(e^{t}\right)^{\ln \left(e^{z}\right)}\right|_{G} \\
& =\left(e^{|t|}\right)^{\ln \left(e^{|z|}\right)}=e^{|t|} \odot e^{|z|}=|u|_{G} \odot|v|_{G}
\end{aligned}
$$


we derive that the equality

$$
|u \odot v|_{G}=|u|_{G} \odot|v|_{G}
$$

holds, as desired.

In (CC), the usual distance is given by $\left|z_{1}-z_{2}\right|=\sqrt{\left(x_{1}-x_{2}\right)^{2}+\left(y_{1}-y_{2}\right)^{2}}$, where $z_{1}, z_{2} \in \mathbb{C}$ with $z_{1}=x_{1}+i y_{1}, z_{2}=x_{2}+i y_{2}$. In (GC), the geometric distance is defined by

$$
\begin{aligned}
\left|u_{1} \ominus u_{2}\right|_{G} & =\sqrt{\left(u_{1} \ominus u_{2}\right)^{2} \oplus \oplus\left(v_{1} \ominus v_{2}\right)^{2} G} G \\
& =e^{\left[\ln \left(\left|\frac{u_{1}}{u_{2}}\right|_{G} \ln \left|\frac{u_{1}}{u_{2}}\right|_{G} \cdot\left|\frac{v_{1}}{v_{2}}\right|_{G}{ }^{\ln \left|\frac{v_{1}}{v_{2}}\right|_{G}}\right)\right]^{1 / 2}},
\end{aligned}
$$

where $u_{1}, u_{2} \in \mathbb{C}(G)$ with $u_{1}=e^{z_{1}}, u_{2}=e^{z_{2}}$ such that

$$
z_{1}=x_{1}+i y_{1}, z_{2}=x_{2}+i y_{2} \in \mathbb{C}_{s t r} .
$$

So $e^{z_{1}}=u_{1}, e^{z_{2}}=u_{2} \in \mathbb{C}(G)$ and $u_{1}=u_{1} \cdot v_{1}^{\ln i}, u_{2}=u_{2} \cdot v_{2}^{\ln i}$ it is clear that $u_{1}=e^{x_{1}}, u_{2}=e^{x_{2}}, v_{1}=e^{y_{1}}, v_{2}=e^{y_{2}}$

We can define another way, replacing each exp function by its corresponding statement.

$$
\begin{aligned}
& \left|u_{1} \ominus u_{2}\right|_{G}={\sqrt{\left(u_{1} \ominus u_{2}\right)^{2_{G}} \oplus\left(v_{1} \ominus v_{2}\right)^{2_{G}}}}^{G} \\
& =e^{\left[\ln \left(\left|\frac{u_{1}}{u_{2}}\right|_{G}^{\ln \left|\frac{u_{1}}{u_{2}}\right|_{G}} \cdot\left|\frac{v_{1}}{v_{2}}\right|_{G} \ln \left|\frac{v_{1}}{v_{2}}\right|_{G}\right)\right]^{1 / 2}}
\end{aligned}
$$

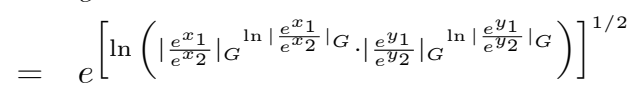

$$
\begin{aligned}
& =e^{\left[\ln \left(\left|e^{x_{1}-x_{2}}\right|_{G}^{\ln \left|e^{x_{1}-x_{2}}\right|_{G}} \cdot\left|e^{y_{1}-y_{2}}\right|_{G}{ }^{\ln \left|e^{y_{1}-y_{2}}\right|_{G}}\right)\right]^{1 / 2}}
\end{aligned}
$$

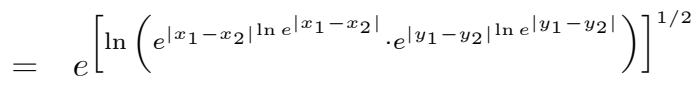

$$
\begin{aligned}
& =e^{\left[\ln \left(e^{\left|x_{1}-x_{2}\right|^{2}} \cdot e^{\left|y_{1}-y_{2}\right|^{2}}\right)\right]^{1 / 2}} \\
& =e^{\left[\ln \left(e^{\left|x_{1}-x_{2}\right|^{2}+\left|y_{1}-y_{2}\right|^{2}}\right)\right]^{1 / 2}} \\
& =e^{\sqrt{\left|x_{1}-x_{2}\right|^{2}+\left|y_{1}-y_{2}\right|^{2}}} \\
& =e^{\sqrt{\left|\ln u_{1}-\ln u_{2}\right|^{2}+\left|\ln v_{1}-\ln v_{2}\right|^{2}}}
\end{aligned}
$$

\section{Geometric Metric Spaces}

In this section, we deal with the vector spaces over the field $\mathbb{C}(G)$ of geometric complex numbers. Prior to giving the theorems on the geometric vector spaces, we prove the required inequalities in the sense of (GC).

Lemma 3.1. [Triangle inequality] Let $x, y \in \mathbb{C}(G)$. Then,

$$
|x \oplus y|_{G} \leq|x|_{G} \oplus|y|_{G} .
$$


Proof. Let $x, y \in \mathbb{C}(G)$ with $x=e^{u}, y=e^{v}$. Then, by bearing in mind the triangle inequality in $(\mathrm{CC})$ one can easily see that

$$
\begin{aligned}
|x \oplus y|_{G} & =\left|e^{u+v}\right|_{G}=e^{|u+v|} \\
& \leq e^{|u|+|v|}=\left|e^{u}\right|_{G} \cdot\left|e^{v}\right|_{G} \\
& =|x|_{G} \oplus|y|_{G},
\end{aligned}
$$

i.e., the geometric triangle inequality in (3.1) holds.

Lemma 3.2. Let $u, v \in \mathbb{C}(G)$. Then,

$$
\frac{|u \oplus v|_{G}}{1 \oplus|u \oplus v|_{G}} G \leq \frac{|u|_{G}}{1 \oplus|u|_{G}} G \oplus \frac{|v|_{G}}{1 \oplus|v|_{G}} G
$$

Proof. For this purpose we use the auxiliary function $f$ defined on $\mathbb{C}(G)$ by $f(t)=t /{ }^{G}(1 \oplus t)$. Geometric derivative $f^{\prime} G$ of $f$ is $f^{\prime} G(t)=e^{\frac{1}{1+t^{2}}}$ which is positive in the sense of (GC). Hence $f$ is monotone increasing. Consequently, the triangle inequality in (3.1) implies that $f\left(|u \oplus v|_{G}\right) \leq f\left(|u|_{G} \oplus|v|_{G}\right)$. Therefore, we have

$$
\begin{aligned}
\frac{|u \oplus v|_{G}}{1 \oplus|u \oplus v|_{G}} G & \leq \frac{|u|_{G} \oplus|v|_{G}}{1 \oplus|u|_{G} \oplus|v|_{G}} G \\
& =\frac{|u|_{G}}{1 \oplus|u|_{G} \oplus|v|_{G}} G \oplus \frac{|v|_{G}}{1 \oplus|u|_{G} \oplus|v|_{G}} G \\
& \leq \frac{|u|_{G}}{1 \oplus|u|_{G}} G \oplus \frac{|v|_{G}}{1 \oplus|v|_{G}} G
\end{aligned}
$$

as desired.

Now, we state and prove the Minkowski's inequality in the sense of (GC).

Lemma 3.3. [Minkowski's inequality] Let $p \geq 1$ and $a_{k}, b_{k} \in \mathbb{C}(G)$ with $a_{k}=e^{c_{k}}$, $b_{k}=e^{d_{k}}$ for $k \in\{1,2,3, \ldots, n\}$. Then,

$$
{\sqrt[p]{G} \sum_{k=1}^{n}\left|a_{k} \oplus b_{k}\right|_{G}^{p_{G}}}^{G} \leq{\sqrt[p]{\sum_{k=1}^{n}\left|a_{k}\right|_{G}^{p_{G}}}}^{G} \oplus{\sqrt[p]{\sum_{k=1}^{n}\left|b_{k}\right|_{G}^{p_{G}}}}^{G} .
$$

Proof. Suppose that $p \geq 1$ and $a_{k}, b_{k} \in \mathbb{C}(G)$ with $a_{k}=e^{c_{k}}, b_{k}=e^{d_{k}}$ for $k \in\{1,2,3, \ldots, n\}$. Then, by taking into account the Minkowski's inequality in the 
sense of (CC) a direct calculation leads us to

$$
\begin{aligned}
\sqrt[p]{\sum_{k=1}^{n}\left|a_{k} \oplus b_{k}\right|_{G}^{p_{G}}} & =e^{\left(\ln \prod_{k=1}^{n}\left|a_{k} \cdot b_{k}\right|^{\ln p-1}\left|a_{k} \cdot b_{k}\right|\right)^{\frac{1}{p}}} \\
& =e^{\left[\ln \prod_{k=1}^{n}\left(e^{\left|c_{k}+d_{k}\right|}\right)^{\left.\ln ^{p-1}\left(e^{\left|c_{k}+d_{k}\right|}\right)\right]^{\frac{1}{p}}}\right.} \\
& =e^{\left[\ln \prod_{k=1}^{n}\left(e^{\left|c_{k}+d_{k}\right|}\right)^{\left|c_{k}+d_{k}\right|^{p-1}}\right]^{\frac{1}{p}}} \\
& =e^{\left[\ln e^{\sum_{k=1}^{n}\left|c_{k}+d_{k}\right|^{p}}\right]^{\frac{1}{p}}} \\
& \leq e^{\left(\sum_{k=1}^{n}\left|c_{k}\right|^{p}\right)^{\frac{1}{p}}+\left(\sum_{k=1}^{n}\left|d_{k}\right|^{p}\right)^{\frac{1}{p}}} \\
& =\sqrt\left[\prod_{k=1}^{n}\left|a_{k}\right|^{\ln ^{p-1}\left|a_{k}\right|}\right) \cdot\left(\prod_{k=1}^{n}\left|b_{k}\right|^{\ln ^{p-1}\left|b_{k}\right|}\right]{\sum_{k=1}^{n}\left|a_{k}\right|_{G}^{p_{G}} \oplus \sqrt[p]{\sum_{k=1}^{n}\left|b_{k}\right|_{G}^{p_{G}}},}
\end{aligned}
$$

as asserted.

Now, we can give the definition of the concepts metric and norm, in the sense of geometric calculus.

Definition 3.4. Let $X$ be any non-empty set. Then, a geometric metric space is a pair $\left(X, d_{G}\right)$, where $d_{G}$ is a geometric metric function $d_{G}: X \times X \rightarrow \mathbb{R}^{+}(G)$ such that the following geometric metric axioms hold:

GM1 $d_{G}(x, y)=1$ if and only if $x=y$,

GM2 $d_{G}(x, y)=d_{G}(y, x)$, (symmetry)

GM3 $d_{G}(x, y) \leq d_{G}(x, z) \oplus d_{G}(z, y)$, (triangle inequality)

for all $x, y, z \in X$.

Definition 3.5. Let $X$ be a vector space over the field $\mathbb{C}(G)$ and $\|\cdot\|_{G}$ be a function from $X$ to $\mathbb{R}^{+}(G)$ satisfying the following properties: For $x, y \in X$ and $\alpha \in \mathbb{C}(G)$,

GN1 $\|x\|_{G}=1$ if and only if $x=\theta_{G}$,

GN2 $\|\alpha x\|_{G}=|\alpha|_{G}\|x\|_{G}$,

GN3 $\|x \oplus y\|_{G} \leq\|x\|_{G} \oplus\|y\|_{G}$, (triangle inequality).

Then, $\left(X,\|\cdot\|_{G}\right)$ is said a geometric normed space.

It is trivial that a geometric norm $\|\cdot\|_{G}$ on $X$ defines a geometric metric $d_{G}$ on $X$ which is given by

$$
d_{G}(x, y)=\|x \ominus y\|_{G} ; \quad(x, y \in X)
$$

and is called the geometric metric induced by the geometric norm. 
It is not hard to show that $\left(\mathbb{R}(G), d_{G}\right)$ is a metric space with the usual metric $d_{G}$ defined by

$$
\begin{aligned}
d_{G}: \mathbb{R}(G) \times \mathbb{R}(G) & \longrightarrow \mathbb{R}^{+}(G) \\
(x, y) & \longmapsto d_{G}(x, y)=|x \ominus y|_{G} .
\end{aligned}
$$

Geometric complex metric: Assuming $z_{1}=x_{1}+i y_{1}, z_{2}=x_{2}+i y_{2} \in \mathbb{C}_{s t r}$ so $e^{z_{1}}=w_{1}, e^{z_{2}}=w_{2} \in \mathbb{C}(G)$ and $w_{1}=u_{1} \oplus i \odot v_{1}, w_{2}=u_{2} \oplus i \odot v_{2}$ it's clear that $u_{1}=e^{x_{1}}, u_{2}=e^{x_{2}}, v_{1}=e^{y_{1}}, v_{2}=e^{y_{2}}$ so we define the metric as follows

$$
\begin{aligned}
d_{G}\left(w_{1}, w_{2}\right) & =\left|w_{1} \ominus w_{2}\right|_{G}=\sqrt{\left(u_{1} \ominus u_{2}\right)^{2} G} \oplus\left(v_{1} \ominus v_{2}\right)^{2_{G}} G \\
& =e^{\sqrt{\left|\ln u_{1}-\ln u_{2}\right|^{2}+\left|\ln v_{1}-\ln v_{2}\right|^{2}}}
\end{aligned}
$$

Theorem 3.6. $n$-dimensional geometric complex Euclidian space $\mathbb{C}^{n}(G)$ consists of all ordered n-tuples of geometric complex numbers is a metric space with the metric $d_{G}$ defined by

$$
\begin{aligned}
d_{G}: \mathbb{C}^{n}(G) \times \mathbb{C}^{n}(G) & \longrightarrow \mathbb{R}^{+}(G) \\
(x, y) & \longmapsto d_{G}(x, y)=\sqrt{{ }_{G} \sum_{k=1}^{n}\left(x_{k} \ominus y_{k}\right)^{2_{G}}} \\
& =e^{\ln \left(\prod_{k=1}^{n}\left|\frac{x_{k}}{y_{k}}\right|_{G}^{\ln \left|\frac{x_{k}}{y_{k}}\right|_{G}}\right)^{1 / 2}}
\end{aligned}
$$

where $x=\left(x_{1}, x_{2}, \ldots, x_{n}\right), y=\left(y_{1}, y_{2}, \ldots, y_{n}\right) \in \mathbb{C}^{n}(G)$.

Proof. One can easily see that the relation $d_{G}$ given by (3.4) satisfies the axioms (GM1) and (GM2). (GM3) also follows from (3.3) with $p=2$, the Cauchy-Schwarz inequality. So, we omit the detail.

\section{Convergence and Completeness in (GC)}

Definition 4.1 (Geometric convergent sequence). A sequence $\left(x_{n}\right)$ in a metric space $\left(X, d_{G}\right)$ is said to be convergent if there is an $x \in X$ such that $\lim _{n \rightarrow \infty} d_{G}\left(x_{n}, x\right)=1$ then $x$ is called the geometric limit of $\left(x_{n}\right)$ and we write

$$
{ }_{n \rightarrow \infty}^{G} \lim _{n}=x \text { or } x_{n} \stackrel{G}{\rightarrow} x, \text { as } n \rightarrow \infty .
$$

Definition 4.2. [Geometric Cauchy sequence] A sequence $\left(x_{n}\right)$ in a geometric metric space $X=\left(X, d_{G}\right)$ is said to be geometric Cauchy if for every $\varepsilon>1$ in $\mathbb{R}^{+}(G)$, there is an $N=N(\varepsilon)$ such that $\left|x_{m} \ominus x_{n}\right|<\varepsilon$ for all $m, n>N$. The space $X$ is said to be geometric complete if every geometric Cauchy sequence converges in $X$.

Theorem 4.3. Every geometric convergent sequence is a geometric Cauchy sequence. 
Proof. Suppose that $\left(x_{n}\right)$ be a geometric convergent sequence with the limit $x$ in a geometric metric space $X=\left(X, d_{G}\right)$. Then, for every $\varepsilon>1$ there is an $N=N(\varepsilon)$ such that $d_{G}\left(x_{n}, x\right)<\frac{\varepsilon}{2} G$ for all $n>N$. Hence by the geometric triangle inequality, we obtain for $m, n>N$ that

$$
d_{G}\left(x_{m}, x_{n}\right) \leq d_{G}\left(x_{m}, x\right) \oplus d_{G}\left(x, x_{n}\right)<\frac{\varepsilon}{2} G \oplus \frac{\varepsilon}{2} G=\varepsilon,
$$

which shows that $\left(x_{n}\right)$ is geometric Cauchy, as asserted.

Theorem 4.4. Let $X=\left(X, d_{G}\right)$ be a geometric metric space. Then, a convergent sequence in $X$ is bounded and its geometric limit is unique.

Proof. Suppose that $\left(x_{n}\right)$ be a convergent sequence in a geometric metric space $X$ with $x_{n} \stackrel{G}{\rightarrow} x$. Then, taking $\varepsilon=e$, we can find an $N$ such that $d_{G}\left(x_{n}, x\right)<e$ for all $n>N$. Hence by the geometric triangle inequality (GM3), for all $n$ we have $d_{G}\left(x_{n}, x\right)<e \oplus a$, where $a=\max \left\{d_{G}\left(x_{1}, x\right), \ldots, d_{G}\left(X_{N}, x\right)\right\}$. This shows that $\left(x_{n}\right)$ is bounded. Assuming that $x_{n} \stackrel{G}{\rightarrow} x$ and $x_{n} \stackrel{G}{\longrightarrow} z$, we obtain from (GM3)

$$
1 \leq d_{G}(x, z) \leq d_{G}\left(x, x_{n}\right) \oplus d_{G}\left(x_{n}, z\right) \stackrel{G}{\rightarrow} 1 \oplus 1=1
$$

and the uniqueness $x=z$ of the geometric limit follows from (GM1).

Now, we can give the theorem on the completeness of the metric space $\left(\mathbb{C}^{n}(G), d_{G}\right)$.

Theorem 4.5. $\left(\mathbb{C}^{n}(G), d_{G}\right)$ is complete.

Proof. It is known by Theorem 3.6 that $d_{G}$ defined by (3.4) is a metric on $\mathbb{C}^{n}(G)$. Suppose that $\left(x_{m}\right)=\left\{x_{k}^{(m)}\right\}$ is a Cauchy sequence in $\mathbb{C}^{n}(G)$, where

$$
x_{m}=\left(x_{1}^{(m)}, x_{2}^{(m)}, x_{3}^{(m)}, \ldots, x_{n}^{(m)}\right)
$$

for each fixed $m \in \mathbb{N}$. Since $\left(x_{m}\right)$ is a geometric Cauchy, for every $\varepsilon>1$ there is an $N \in \mathbb{N}$ such that

$$
d_{G}\left(x_{m}, x_{r}\right)={\sqrt{\sum_{k=1}^{n}\left(x_{k}^{(m)} \ominus x_{k}^{(r)}\right)^{2_{G}}}}^{G}<\varepsilon \text { for all } m, r>N .
$$

Geometric squaring, we have for all $m, r>N$ and all $k \in\{1,2,3, \ldots, n\}$ that

$$
\left(x_{k}^{(m)} \ominus x_{k}^{(r)}\right)^{2_{G}}<\varepsilon^{2_{G}}
$$

which is equivalent to

$$
\left|x_{k}^{(m)} \ominus x_{k}^{(r)}\right|_{G}<\varepsilon .
$$

This shows that for each fixed $k \in\{1,2, \ldots, n\},\left(x_{k}^{(1)}, x_{k}^{(2)}, x_{k}^{(3)}, \ldots\right)$ is a convergent sequence with $x_{k}^{(m)} \stackrel{G}{\rightarrow} x_{k}$, as $m \rightarrow \infty$. Using these $n$ limits, we define $x=\left(x_{1}, x_{2}, x_{3}, \ldots, x_{n}\right)$ in $\mathbb{C}^{n}(G)$. From (4.1), letting $r \rightarrow \infty$ it is obtained that 
$d_{G}\left(x_{m}, x\right) \leq \varepsilon$ for all $m>N$ which shows that $\left(x_{m}\right)$ converges in $\mathbb{C}^{n}(G)$. Consequently, $\left(\mathbb{C}^{n}(G), d_{G}\right)$ is a complete metric space.

Since it is known that $\mathbb{C}^{n}(G)$ is a complete metric space with the metric $d_{G}$ defined by (3.4) induced by the norm $\|\cdot\|_{G}$, as a direct consequence of Theorem 4.5, we have:

Corollary 1. $\mathbb{C}^{n}(G)$ is a Banach space with the norm $\|\cdot\|_{G}$ defined by

$$
\|x\|_{G}=\sqrt{\sum_{k=1}^{n}\left|x_{k}\right|_{G}^{2_{G}}}, x=\left(x_{1}, x_{2}, \ldots, x_{n}\right) \in \mathbb{C}^{n}(G) .
$$

\section{Sequence Spaces in the Field $\mathbb{C}(G)$}

In this section, we define the sets $\omega(G), \ell_{\infty}(G), c(G), c_{0}(G)$ and $\ell_{p}(G)$ of all, bounded, convergent, null and absolutely $p$-summable sequences over the geometric complex field $\mathbb{C}(G)$ which correspond to the sets $\omega, \ell_{\infty}, c, c_{0}$ and $\ell_{p}$ over the complex field $\mathbb{C}$, respectively. That is to say that

$$
\begin{aligned}
\omega(G) & =\left\{x=\left(x_{k}\right): x_{k} \in \mathbb{C}(G) \text { for all } k \in \mathbb{N}\right\}, \\
\ell_{\infty}(G) & =\left\{x=\left(x_{k}\right) \in \omega(G): \sup _{k \in \mathbb{N}}\left|x_{k}\right|_{G}<\infty\right\}, \\
c(G) & =\left\{x=\left(x_{k}\right) \in \omega(G):{ }_{k \rightarrow \infty}^{G} \lim _{k \rightarrow \infty}\left|x_{k} \ominus \ell\right|_{G}=1\right\}, \\
c_{0}(G) & =\left\{x=\left(x_{k}\right) \in \omega(G):{ }_{k \rightarrow \infty}^{G} \lim _{k \rightarrow \infty} x_{k}\right\}, \\
\ell_{p}(G) & =\left\{x=\left(x_{k}\right) \in \omega(G):{ }_{G} \sum_{k=0}^{\infty}\left|x_{k}\right|_{G}^{p_{G}}<\infty\right\} .
\end{aligned}
$$

One can easily see that the set $\omega(G)$ forms a vector space over $\mathbb{C}(G)$ with respect to the algebraic operations $\oplus$ addition and $\odot$ scalar multiplication defined on $\omega(G)$, as follows:

$$
\begin{aligned}
\oplus: \omega(G) \times \omega(G) & \longrightarrow \omega(G) \\
(x, y) & \longmapsto x \oplus y=\left(x_{k}\right) \oplus\left(y_{k}\right)=\left(x_{k} y_{k}\right), \\
\odot: \mathbb{C}(G) \times \omega(G) & \longrightarrow \omega(G) \\
(\alpha, y) & \longmapsto \alpha \odot y=\alpha \odot\left(y_{k}\right)=\left(\alpha^{\ln y_{k}}\right),
\end{aligned}
$$

where $x=\left(x_{k}\right), y=\left(y_{k}\right) \in \omega(G)$ and $\alpha \in \mathbb{C}(G)$. It is not hard to show that the sets $\ell_{\infty}(G), c(G), c_{0}(G)$ and $\ell_{p}(G)$ are the subspaces of the space $\omega(G)$. This means that $\ell_{\infty}(G), c(G), c_{0}(G)$ and $\ell_{p}(G)$ are the classical sequence spaces over the field $\mathbb{C}(G)$. 
Theorem 5.1. Define $d_{G}$ on the space $\omega(G)$ by

$$
d_{G}(x, y)=\sum_{G=1}^{\infty} \frac{\left|x_{k} \ominus y_{k}\right|_{G}}{2^{k_{G}} \odot\left(1 \oplus\left|x_{k} \ominus y_{k}\right|_{G}\right)} G,
$$

where $x=\left(x_{k}\right), y=\left(y_{k}\right) \in \omega(G)$. Then, $\left(\omega(G), d_{G}\right)$ is a metric space.

Proof. Since the axioms (GM1) and (GM2) are easily satisfied, we omit the detail. Since the inequality

$$
\frac{\left|x_{k} \ominus y_{k}\right|_{G}}{1 \oplus\left|x_{k} \ominus y_{k}\right|_{G}} G \leq \frac{\left|x_{k} \ominus z_{k}\right|_{G}}{1 \oplus\left|x_{k} \ominus z_{k}\right|_{G}} G \oplus \frac{\left|z_{k} \ominus y_{k}\right|_{G}}{1 \oplus\left|z_{k} \ominus y_{k}\right|_{G}} G
$$

holds by(3.2) for all $x_{k}, y_{k}, z_{k} \in \mathbb{C}(G)$, multiplying the inequality (5.1) by $\frac{1}{2^{k} G} G$ and nextly summing over $1 \leq k \leq n$ one can derive that

$$
\begin{aligned}
& \sum_{k=1}^{n} \frac{\left|x_{k} \ominus y_{k}\right|_{G}}{2^{k_{G}} \odot\left(1 \oplus\left|x_{k} \ominus y_{k}\right|_{G}\right)} G \\
& \leq \sum_{G=1}^{n} \frac{\left|x_{k} \ominus z_{k}\right|_{G}}{2^{k_{G}} \odot\left(1 \oplus\left|x_{k} \ominus z_{k}\right|_{G}\right)} G \oplus \sum_{G=1}^{n} \frac{\left|z_{k} \ominus y_{k}\right|_{G}}{2^{k_{G}} \odot\left(1 \oplus\left|z_{k} \ominus y_{k}\right|_{G}\right)} G .
\end{aligned}
$$

Additionally, since the inequalities

$$
\begin{aligned}
\frac{\left|x_{k} \ominus y_{k}\right|_{G}}{2^{k_{G}} \odot\left(1 \oplus\left|x_{k} \ominus y_{k}\right|_{G}\right)} G & \leq \frac{1}{2^{k_{G}}} G, \\
\frac{\left|x_{k} \ominus z_{k}\right|_{G}}{2^{k_{G}} \odot\left(1 \oplus\left|x_{k} \ominus z_{k}\right|_{G}\right)} G & \leq \frac{1}{2^{k_{G}}} G, \\
\frac{\left|z_{k} \ominus y_{k}\right|_{G}}{2^{k_{G}} \odot\left(1 \oplus\left|z_{k} \ominus y_{k}\right|_{G}\right)} G & \leq \frac{1}{2^{k_{G}}} G
\end{aligned}
$$

hold for all $k \in \mathbb{N}$, the comparison test yields that the series

$$
\begin{aligned}
& \sum_{k=1}^{\infty} \frac{\left|x_{k} \ominus y_{k}\right|_{G}}{2^{k_{G}} \odot\left(1 \oplus\left|x_{k} \ominus y_{k}\right|_{G}\right)} G, \\
& \sum_{G=1}^{\infty} \frac{\left|x_{k} \ominus z_{k}\right|_{G}}{2^{k_{G}} \odot\left(1 \oplus\left|x_{k} \ominus z_{k}\right|_{G}\right)} G, \\
& \sum_{G=1}^{\infty} \frac{\left|z_{k} \ominus y_{k}\right|_{G}}{2^{k_{G}} \odot\left(1 \oplus\left|z_{k} \ominus y_{k}\right|_{G}\right)} G \text {. }
\end{aligned}
$$


are geometric convergent. Therefore, by letting $n \rightarrow \infty$ in (5.2) that

$$
\begin{aligned}
d_{G}(x, y) & =\sum_{G=1}^{\infty} \frac{\left|x_{k} \ominus y_{k}\right|_{G}}{2^{k_{G}} \odot\left(1 \oplus\left|x_{k} \ominus y_{k}\right|_{G}\right)} G \\
& \leq \sum_{k=1}^{\infty} \frac{\left|x_{k} \ominus z_{k}\right|_{G}}{2^{k_{G}} \odot\left(1 \oplus\left|x_{k} \ominus z_{k}\right|_{G}\right)} G \oplus \sum_{G}^{\infty} \frac{\left|z_{k} \ominus y_{k}\right|_{G}}{2^{k_{G}} \odot\left(1 \oplus\left|z_{k} \ominus y_{k}\right|_{G}\right)} G \\
& =d_{G}(x, z) \oplus d_{G}(z, y),
\end{aligned}
$$

i.e., (GM3) holds. This step completes the proof.

Theorem 5.2. The inclusion $\omega(G) \subset \omega$ strictly holds.

Proof. Let $x=\left(x_{k}\right) \in \omega(G)$ with $x_{k}=e^{y_{k}}$ for all $k \in \mathbb{N}$. Then, $x \in \omega$ which shows that $\omega(G) \subset \omega$. Although $y=\left(y_{k}\right)=(0,0,0, \ldots)=\theta \in \omega, \theta \notin \omega(G)$ since $x_{k}=e^{y_{k}} \neq 0$ for all $k \in \mathbb{N}$. Hence, the inclusion $\omega(G) \subset \omega$ is strict.

Theorem 5.3. Define $d_{G_{\infty}}$ on the geometric space $\lambda(G)$ by

$$
d_{G_{\infty}}(x, y)=\sup _{k \in \mathbb{N}}\left|x_{k} \ominus y_{k}\right|_{G}
$$

where $\lambda$ denotes any of the spaces $\ell_{\infty}, c$ and $c_{0}$, and $x=\left(x_{k}\right), y=\left(y_{k}\right) \in \lambda(G)$. Then, $\left(\lambda(G), d_{G_{\infty}}\right)$ is a complete metric space.

Proof. Since the proof is similar for the spaces $c(G)$ and $c_{0}(G)$, we prove the theorem only for the space $\ell_{\infty}(G)$. Let $x=\left(x_{k}\right), y=\left(y_{k}\right), z=\left(z_{k}\right) \in \ell_{\infty}(G)$. Then,

(i) Suppose that $d_{G_{\infty}}(x, y)=1$. This implies for all $k \in \mathbb{N}$ that $x_{k} \ominus y_{k}=x_{k} / y_{k}=1$, i.e., $x=y$.

Conversely, let $x=y$. Then, we have $x_{k} / y_{k}=x_{k} \ominus y_{k}=1$ which yields that $d_{G_{\infty}}(x, y)=1$.

That is to say that (GM1) holds.

(ii) It is immediate that $d_{G_{\infty}}(x, y)=d_{G_{\infty}}(y, x)$, i.e., (GM2) holds.

(iii) By taking into account the triangle inequality in (3.1) we see that

$$
\begin{aligned}
d_{G_{\infty}}(x, y) & =\sup _{k \in \mathbb{N}}\left|x_{k} \ominus y_{k}\right|_{G} \\
& =\sup _{k \in \mathbb{N}}\left|x_{k} \ominus y_{k} \oplus z_{k} \ominus z_{k}\right|_{G} \\
& \leq \sup _{k \in \mathbb{N}}\left|x_{k} \ominus z_{k}\right|_{G} \oplus \sup _{k \in \mathbb{N}}\left|y_{k} \ominus z_{k}\right|_{G} \\
& =d_{G_{\infty}}(x, z)+d_{G_{\infty}}(z, y) .
\end{aligned}
$$

Hence, (GM3) is satisfied.

Since the axioms (GM1)-(GM3) are satisfied, $\left(\ell_{\infty}(G), d_{G_{\infty}}\right)$ is a metric space.

It remains to prove the completeness of the space $\ell_{\infty}(G)$. Let $\left(x^{m}\right)$ be any Cauchy sequence in the space $\ell_{\infty}(G)$, where $x^{m}=\left\{x_{1}^{(m)}, x_{2}^{(m)}, \ldots\right\}$. Then, for any 
$\varepsilon>1$ there is an $N$ such that for all $m, n>N$,

$$
d_{G_{\infty}}\left(x^{m}, x^{n}\right)=\sup _{k \in \mathbb{N}}\left|x_{k}^{(m)} \ominus x_{k}^{(n)}\right|_{G}<\varepsilon .
$$

A fortiori, for every fixed $k \in \mathbb{N}$ and for $m, n>N$

$$
\left|x_{k}^{(m)} \ominus x_{k}^{(n)}\right|_{G}<\varepsilon .
$$

Hence for every fixed $k \in \mathbb{N}$, the sequence $x_{m}=\left\{x_{k}^{(1)}, x_{k}^{(2)}, \ldots\right\}$ is a Cauchy sequence of geometric complex numbers. Since $\mathbb{C}(G)$ is a complete metric space by Theorem 4.5 with $n=1$, it converges, say $x_{k}^{(m)} \rightarrow x_{k}$ as $m \rightarrow \infty$. Using these infinitely many limits $x_{1}, x_{2}, \ldots$, we define $x=\left(x_{1}, x_{2}, \ldots\right)$ and show that $x \in \ell_{\infty}(G)$. From (5.3) letting $n \rightarrow \infty$ and $m>N$ we have

$$
\left|x_{k}^{(m)} \ominus x_{k}\right|_{G} \leq \varepsilon \text {. }
$$

Since $\left(x^{m}\right)=\left(x_{k}^{(m)}\right) \in \ell_{\infty}(G)$, there is a geometric real number $k_{m}$ such that $\left|x_{k}^{(m)}\right|_{G} \leq k_{m}$ for all $k \in \mathbb{N}$. Thus, (5.4) gives together with the triangle inequality in (3.1) for $m>N$ that

$$
\left|x_{k}\right|_{G} \leq\left|x_{k} \ominus x_{k}^{(m)}\right|_{G}+\left|x_{k}^{(m)}\right|_{G} \leq \varepsilon+k_{m} .
$$

It is clear that (5.5) holds for every $k \in \mathbb{N}$ whose right-hand side does not involve $k$. Hence $\left(x_{k}\right)$ is a bounded sequence of geometric complex numbers, i.e., $x=\left(x_{k}\right) \in \ell_{\infty}(G)$. Also from (5.4) we obtain for $m>N$ that

$$
d_{G_{\infty}}\left(x^{m}, x\right)=\sup _{k \in \mathbb{N}}\left|x_{k}^{(m)} \ominus x_{k}\right|_{G} \leq \varepsilon .
$$

This shows that $x^{m} \stackrel{G}{\rightarrow} x$, as $m \rightarrow \infty$. Since $\left(x^{m}\right)$ is an arbitrary geometric Cauchy sequence, $\ell_{\infty}(G)$ is complete.

Since it is known by Theorem 5.3 that the spaces $\ell_{\infty}(G), c(G)$ and $c_{0}(G)$ are the complete metric spaces with the metric $d_{G_{\infty}}$ induced by the norm $\|\cdot\|_{G_{\infty}}$, we have:

Corollary 2. $\ell_{\infty}(G), c(G)$ and $c_{0}(G)$ are the Banach spaces with the norm $\|\cdot\|_{G_{\infty}}$ defined by

$$
\|x\|_{G_{\infty}}=\sup _{k \in \mathbb{N}}\left|x_{k}\right|_{G} ; x=\left(x_{1}, x_{2}, \ldots, x_{n}\right) \in \lambda(G), \lambda \in\left\{\ell_{\infty}, c, c_{0}\right\} .
$$

To avoid undue repetition in the statements, we give the final theorem is on the complete metric space $\ell_{p}(G)$ without proof. 
Theorem 5.4. Let $d_{G_{p}}$ be defined on the space $\ell_{p}(G)$ by

$$
d_{G_{p}}(x, y)=\left({ }_{G} \sum_{k=0}^{\infty}\left|x_{k} \ominus y_{k}\right|_{G}^{p_{G}}\right)^{(1 \oslash p)_{G}}, \text { where } x=\left(x_{k}\right), y=\left(y_{k}\right) \in \ell_{p}(G) .
$$

Then, $\left(\ell_{p}(G), d_{G_{p}}\right)$ is a complete metric space.

Theorem 5.5. $\ell_{p}(G)$ is a Banach space with the norm $\|\cdot\|_{G_{p}}$ defined by

$$
\|x\|_{G_{p}}=\left({ }_{G} \sum_{k=0}^{\infty}\left|x_{k}\right|_{G}^{p_{G}}\right)^{(1 \oslash p)_{G}}, x=\left(x_{1}, x_{2}, \ldots, x_{n}\right) \in \ell_{p}(G) .
$$

\section{Conclusion}

Some of the analogies between (CC) and the (GC) are demonstrated by theoretical examples. Some important inequalities such as triangle, Minkowski and some other inequalities in the sense of (GC) which are frequently used, are proved. We derive classical sequence spaces in the sense of geometric calculus and try to understand their structure of being geometric vector space. Generally we work on vector spaces which concern physics and computing. There are lots of techniques that have been developed in the sense of the (CC). If the (GC) is employed together with the $(\mathrm{CC})$ in the formulations, then many of the complicated phenomena in physics or engineering may be analyzed more easily.

Quite recently, Talo and Başar have studied the certain sets of sequences of fuzzy numbers and introduced the classical sets $\ell_{\infty}(F), c(F), c_{0}(F)$ and $\ell_{p}(F)$ consisting of the bounded, convergent, null and absolutely $p$-summable sequences of fuzzy numbers in [6]. Nextly, they have defined the $\alpha$-, $\beta$ - and $\gamma$-duals of a set of sequences of fuzzy numbers, and gave the duals of the classical sets of sequences of fuzzy numbers together with the characterization of the classes of infinite matrices of fuzzy numbers transforming one of the classical set into another one. Following Bashirov et al. [1] and Uzer [7], we have given the corresponding results for geometric calculus to the results derived for fuzzy valued sequences in Talo and Başar [6], as a beginning. As a natural continuation of this paper, we should record from now on that it is meaningful to define the $\alpha$-, $\beta$ - and $\gamma$-duals of a set of sequences of geometric complex numbers, and to determine the duals of classical spaces $\ell_{\infty}(G)$, $c(G), c_{0}(G)$ and $\ell_{p}(G)$. Further, one can obtain the similar results by using another type of calculus instead of geometric calculus.

\section{ACKNOWLEDGEMENTS}

The authors wish to express their pleasure to Professor Agamirza E. Bashirov, Department of Mathematics, Eastern Mediterranean University, Gazimagusa - North Cyprus, via Mersin 10 - Turkey, for his careful reading of an earlier version of this paper and constructive suggestions which led to some improvements of the paper. 
The authors also would like to thank Professor Ali Uzer, Department of Electrical and Electronics Engineering, Fatih University, 34500 Büyükçekmece İstanbul, TURKEY, for his making some useful comments which improved the presentation of the paper.

Özet: Klâsik hesap tarzına bir alternatif olarak Grossman ve Katz, [Non-Newtonian Calculus, Lee Press, Pigeon Cove, Massachusetts, 1972] künyeli eserlerinde geometrik, anageometrik ve bigeometrik adlı çeşitleriyle Newtonyen olmayan hesap tarzını literatüre sunmuşlardı. Bu çalışmada; Grossman ve Katz takip edilerek geometrik kompleks sayıların $\mathbb{C}(G)$ cismi ve geometrik metrik kavramı inşa edilmiştir. Daha sonra geometrik hesap tarzına göre üçgen ve Minkowski eşitsizlikleri ispat edilerek geometrik kompleks değerli bütün dizilerin $\omega(G)$, sınırlı dizilerin $\ell_{\infty}(G)$, yakınsak dizilerin $c(G)$, sifira yakınsayan dizilerin $c_{0}(G)$ ve $p$-mutlak toplanabilen dizilerin $\ell_{p}(G)$ cümlelerinin $\mathbb{C}(G)$ cismi üzerinde birer vektör uzayı teşkil ettikleri ve üzerlerinde tanımlanan metriklere göre birer tam metrik uzay oldukları gösterilmiştir.

\section{REFERENCES}

[1] A.E. Bashirov, E.M. Kurpınar, A. Özyapıc1, Multiplicative calculus and its applications, J. Math. Anal. Appl. 337(2008), 36-48.

[2] A. Bashirov, M. R iza, On complex multiplicative differentiation, TWMS J. App. Eng. Math. $1(1)(2011), 75-85$.

[3] A.E. Bashirov, E. Mısırlı, Y. Tandoğdu, A. Özyapıcı, On modeling with multiplicative differential equations, Appl. Math. J. Chinese Univ. 26(4)(2011), 425-438.

[4] A.F. Çakmak, F. Başar, On the classical sequence spaces and non-Newtonian calculus, J. Inequal. Appl. 2012, Art. ID 932734, 13 pp.

[5] M. Grossman, R. Katz, Non-Newtonian Calculus, Lowell Technological Institute, 1972.

[6] Ö. Talo, F. Başar, Determination of the duals of classical sets of sequences of fuzzy numbers and related matrix transformations, Comput. Math. Appl. 58(2009), 717-733.

[7] A. Uzer, Multiplicative type complex calculus as an alternative to the classical calculus, Comput. Math. Appl. 60(2010), 2725-2737.

Current address: Cengiz Türkmen: The Graduate School of Sciences and Engineering, Fatih University, The Hadımköy Campus, Büyükçekmece, 34500-İstanbul, TURKEY

Feyzi Başar: Fatih University, Faculty of Art and Sciences, Department of Mathematics, The Hadımköy Campus, Büyükçekmece, 34500-İstanbul, TURKEY

E-mail address: cturkmen10@gmail.com, fbasar@fatih.edu.tr, feyzibasar@gmail.com

$U R L:$ http://communications.science. ankara.edu.tr/index.php?series $=\mathrm{A} 1$ 